\section{Lesión tumoral sangrante en urgencias: a propósito de un caso de carcinoma mamario en un hombre}

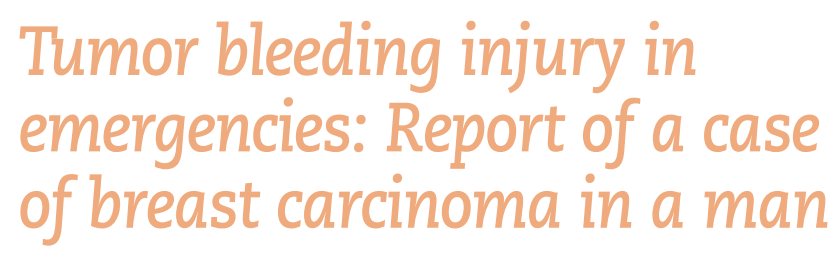

Eva Tizón Bouza ${ }^{1}$

Pilar García Vázquez

Almudena Mato Iglesias²

Miriam Paula Marcos Espino ${ }^{3}$

1. Enfermera. Complejo Hospitalario Universitario de Ferrol. A Coruña (SERGAS).

2. Licenciada en Medicina. Residente de Cirugía General. Complejo Hospitalario Universitario de Ferrol. A Coruña.

3. Enfermera. Servicio Canario de Salud. Las Palmas de Gran Canaria.

\section{Correspondencia:}

Eva Tizón Bouzo

Complejo Hospitalario Universitario de Ferrol

Avenida de la Residencia, s/n

15405 Ferrol (A Coruña)

Tel.: 981334000

E-mail: eva.tizon.bouza@sergas.es

\section{RESUMEN}

El cáncer de mama masculino es una patología poco frecuente y particular que suele afectar a hombres de más 60 años. El presente trabajo tiene como objetivo establecer un plan de trabajo enfermero, centrado en el cuidado de un hombre que presenta una lesión sangrante en una mama, durante su asistencia al Servicio de Urgencias Hospitalarias.

Desarrollaremos el caso a través de la valoración de enfermería según el modelo de Virginia Henderson, los principales diagnósticos, criterios de resultados e intervenciones realizadas.

Una vez estabilizado el sangrado de la herida, se realizan las pruebas pertinentes para diagnosticar al paciente un cáncer de mama derecha en estadio avanzado; se opta por tratamiento paliativo, sin olvidar que los pacientes que reciben estos cuidados buscan preservar la mayor calidad de vida posible y tener una muerte digna.

PALABRAS CLAVE: hemorragia, urgencias médicas, neoplasias de mama, atención de enfermería.

\section{ABSTRACT}

Male breast cancer is a rare disease and usually affects men over 60 years. This paper aims to establish a work plan nurse focus on care for a man who has a bleeding lesion in right breast, while attending to the Emergency Room.

We develop the case through nursing assessment on the model of Virginia Henderson, using a nursing diagnosis, outcome criteria and interventions.

Stabilized the bleeding from the wound, relevant tests are performed to diagnose the patient a right breast cancer in advanced stage.

Palliative treatment is chosen, keeping in mind that patients receiving this care seek to preserve the highest quality of life possible and have dignified death.

KEYWORDS: Hemorrhage, emergencies, breast neoplasms, nursing care.

\section{I) INTRODUCCIÓN}

El cáncer de mama masculino es una patología poco frecuente $(1,2)$. El número de casos de carcinomas de mama que se presentan en hombres es inferior al 1\%, y sólo el $1 \%$ de los diagnósticos de cáncer de mama en el mundo (2). El diagnóstico en los hombres tiene varias particularidades relacionadas con la edad, las comorbilidades, el volumen de la mama, el diagnóstico más tardío y el estadio más avanzado al diagnóstico (por la ausencia de un programa efectivo de cribado en varones) (3). La mediana de edad en el momento del diagnóstico es de 60 a 70 años, aunque este cáncer puede afectar a hombres de todas las edades (4). Mundialmente, el carcinoma de mama masculino se presenta con menor incidencia en la población japonesa, y la mayor, en la raza negra $(5,6)$. Los datos de los que se dispone sobre el cáncer de mama en varones provienen de series pequeñas de pacientes a causa de esta baja prevalencia, por lo que los tratamientos y consideraciones sobre el cáncer de mama en hombres se basan mayoritariamente en protocolos establecidos para las mujeres, aunque debemos tener en cuenta que hay diferencias (2).
Diversos factores de riesgo pueden estar implicados en el desarrollo del carcinoma mamario masculino. Entre ellos destacan los niveles elevados de estradiol y otras hormonas estrogénicas debido a anormalidades testiculares o alteraciones genéticas como el síndrome de Klinefelter $(7,8)$. La concurrencia con algunos antecedentes familiares de carcinoma de la glándula mamaria multiplican hasta por 2,5 veces el riesgo de que un hombre de la familia pueda padecer carcinoma mamario. Otros factores como el alcoholismo, la enfermedad hepática, la obesidad y la dieta han sido propuestos como factores de riesgo, pero la relación ha sido inconstante según diversas investigaciones $(9,10)$. Por último, también se deben tener en cuenta otros factores de riesgo descritos, como la raza (más frecuente en negros), la exposición a radiaciones, altas temperaturas ambientales, la obesidad y la exposición a estrógenos (2).

El diagnóstico en los hombres tiene varias particularidades según la etiología anteriormente planteada. A diferencia de las mujeres, la incidencia del cáncer de mama en los hombres aumenta con la edad; presenta un pico de incidencia a los 75 ańos y la media de edad en el momento del diagnóstico es de 67 años (frente a los 61 años en el caso de las mujeres). 


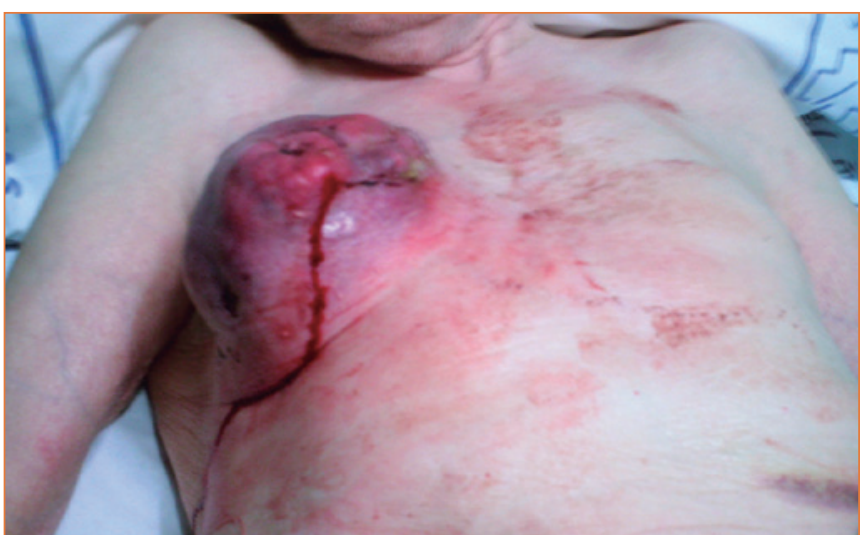

Figura 1. Lesión tumoral sangrante en carcinoma mamario masculino (I).

El diagnóstico más tardío se asocia a peor pronóstico por las comorbilidades asociadas y también por diagnóstico de estadios más avanzados en los hombres (tumores de más de $2 \mathrm{~cm}$ y con ganglios axilares positivos), aunque las variables biológicas de mal pronóstico (receptores hormonales negativos o tumores de alto grado) son más frecuentes en las mujeres (5). En lo referente a las manifestaciones clínicas, la forma más común del carcinoma mamario en hombres presenta masa subareolar no dolorosa, retracción del pezón y secreción hemática (5). Se presenta como un nódulo generalmente en la región areolar o la inversión del pezón, lo que a menudo refleja un estadio avanzado, con un $40 \%$ de los diagnósticos en estadio III o IV (5).

La mayoría de los tumores invasivos en el cáncer de mama en hombres son de tipo ductal por la falta de lobulillos terminales en la mama del hombre. Otras formas de tumores invasivos descritos en hombres son los medulares, tubulares y mucinosos, aunque con menor frecuencia. Los carcinomas inflamatorios y la enfermedad de Paget pueden aparecer y tienen frecuencias similares a las observadas en mujeres (3).

El tejido mamario en el hombre es rudimentario y, generalmente, no se diferencia en la formación de lóbulos terminales a menos que esté expuesto a altas concentraciones endógenas o exógenas de estrógenos. Por esta razón, al igual que en su contraparte femenina, el carcinoma ductal infiltrante (11) es, desde el punto de vista histológico, el subtipo más común, dando cuenta del $90 \%$ de los casos. El carcinoma lobular es raro y ha sido informado no solamente asociado al síndrome de Klinefelter, sino también en pacientes genotípicamente normales y sin exposición previa a estrógenos o historia de ginecomastia $(11,12)$.

Las neoplasias mesenquimatosas benignas de la mama masculina constituyen un grupo heterogéneo e infrecuente de lesiones, la mayor parte de las cuales corresponde a miofibroblastoma $(13,14)$. También se han descrito otras neoplasias, como el tumor fibroso solitario, el tumor de células granulares, el leiomioma o los tumores de origen neurógeno, como el schwannoma, que pueden ser causa de problemas en el diagnóstico diferencial (15). El carcinoma inflamatorio y la enfermedad de Paget del pezón también se han observado en hombres. Se ha descrito también el cáncer intraductal, pero no el carcinoma lobular in situ (11). Los planteamientos terapéuticos se basan en los régimenes aceptados para el cáncer de mama en mujeres, pero hay que tener en cuenta las diferencias entre ambos sexos para proporcionar un mejor tratamiento a estos pacientes (2).

El tratamiento primario estándar consiste en una mastectomía radical modificada con disección axilar $(16,17)$, con biopsia selectiva de ganglio centinela o linfadenectomía; pero las indicaciones de cirugía, así como de radioterapia, hormonoterapia o quimioterapia van a depender del tipo de tumor, de lo avanzado del estadio y de las comorbilidades del paciente (2).

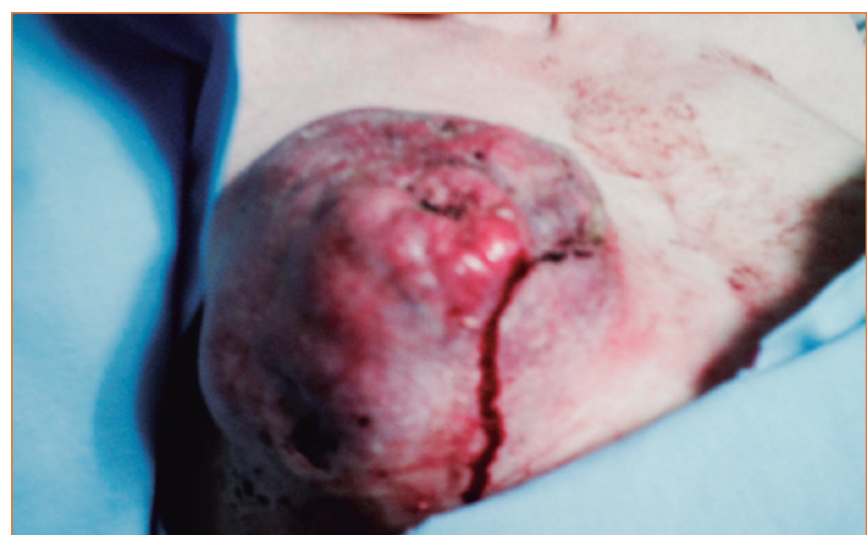

Figura 2. Lesión tumoral sangrante en carcinoma mamario masculino (II).

En los casos de los hombres con tumores con ganglios negativos, se debe considerar la terapia adyuvante (1). En el caso de presencia de ganglios positivos, se utiliza quimioterapia más tamoxifeno y otra terapia hormonal, lo que puede aumentar la supervivencia en el mismo grado que en las mujeres con cáncer de mama. En la actualidad, no hay estudios controlados que comparen las opciones de tratamiento adyuvante.

En caso de enfermedad recidivante localizada, se recomienda la escisión quirúrgica o la radioterapia combinada con quimioterapia (16). Las respuestas son generalmente similares a las observadas en mujeres con cáncer de mama. Sin embargo, en caso de presentar metástasis a distancia, se utiliza terapia hormonal, quimioterapia o una combinación de ambas con cierto éxito. Inicialmente, se recomienda la terapia hormonal (4).

El objetivo de nuestro trabajo es establecer un plan de trabajo enfermero centrado en el cuidado de un hombre que presenta una lesión sangrante en una mama, durante su asistencia al Servicio de Urgencias Hospitalarias.

\section{(0) OBSERVACIÓN Y DESARROLLO DEL PLAN DE ACTUACIÓN}

Descripción del caso clínico de un hombre de 79 años, José Antonio, que acude a las 9 horas de la mañana al Servicio de Urgencias Hospitalarias por sangrado en una herida que presenta en su mama derecha. Ingresa en el servicio de Admisión de Urgencias Hospitalarias por sangrado de una herida en el hemitórax derecho de varias horas de evolución. Se acuesta en una camilla desde triaje de enfermería. Se establece el siguiente diagrama de flujo: Herida, a través del programa de triaje Manchester de implantación en nuestro hospital, se clasifica con una prioridad de atención médica de nivel 4 (verde) de máximo 2 horas para la primera atención médica.

\section{G VALORACIÓN DE ENFERMERÍA}

José Antonio vive desde hace un mes con su hijo de 38 años y la mujer de éste; según refiere el hijo, no tiene alergias de tipo medicamentoso ni alimenticias conocidas. Presenta como antecedentes personales hipertensión arterial y enfermedad de Alzheimer en estadio inicial, aunque José Antonio es independiente para las actividades de la vida diaria (AVD). El tratamiento médico pautado es administrado de manera correcta por su familia según las órdenes médicas; por otro lado, se encuentra vacunado del neumococo y de la gripe. 
Tabla 1. Distribución de clasificaciones de diferentes escalas de valoración de enfermería

\begin{tabular}{|c|c|c|}
\hline Escala de valoración & Valores máximos y mínimos posibles & Puntuación del paciente \\
\hline $\begin{array}{l}\text { Barthel: mide la autonomía para las actividades } \\
\text { de la vida diaria }\end{array}$ & $\begin{array}{l}\text { Máximo: } 100 \text { puntos, independencia } \\
\text { Mínimo: } 0 \text { puntos, dependencia total }\end{array}$ & 60: leve \\
\hline $\begin{array}{l}\text { Karnofsky: mide la calidad de vida de las personas que } \\
\text { padecen cáncer. A mayor grado, mayor calidad de vida }\end{array}$ & $\begin{array}{l}\text { Máximo: } 100 \text { grados, actividad normal. Sin evidencia de enfermedad } \\
\text { Mínimo: } 0 \text { grados, fallecido }\end{array}$ & $\begin{array}{l}\text { 70: cuida de sí mismo pero es incapaz de } \\
\text { Ilevar a cabo una actividad o trabajo normal }\end{array}$ \\
\hline $\begin{array}{l}\text { Escala Palliative Performance Status (21): mide el pronóstico } \\
\text { de supervivencia de los pacientes con cáncer avanzado }\end{array}$ & $\begin{array}{l}\text { Máximo: } 100 \% \text {, normal } \\
\text { Mínimo: } 0 \% \text {, fallecido }\end{array}$ & $\begin{array}{l}\text { 70\%: imposible trabajar, presenta alguna } \\
\text { evidencia de enfermedad }\end{array}$ \\
\hline $\begin{array}{l}\text { Escala SPMSQ de Pfeiffer: mide el deterioro cognitivo. Cuan- } \\
\text { tos más errores, mayor deterioro cognitivo }\end{array}$ & $\begin{array}{l}\text { Máximo: 8-10 errores, deterioro grave } \\
\text { Mínimo: 0-2 errores, normal }\end{array}$ & 3-4 errores: deterioro cognitivo leve \\
\hline $\begin{array}{l}\text { ESCALA de Braden-Bergstrom: mide el riesgo de padecer una } \\
\text { úlcera por presión (UPP) }\end{array}$ & $\begin{array}{l}\text { Máximo: > } 14 \text { puntos, bajo riesgo } \\
\text { Mínimo: }<13 \text { puntos, alto riesgo }\end{array}$ & $14>$, bajo riesgo \\
\hline $\begin{array}{l}\text { Escala visual analógica del dolor (EVA): mide la intensidad del } \\
\text { dolor que sufre una persona }\end{array}$ & $\begin{array}{l}\text { Máximo: } 10 \text {, dolor insoportable } \\
\text { Mínimo: } 0 \text {, ausencia de dolor }\end{array}$ & 3: dolor ligero \\
\hline
\end{tabular}

Se realiza una valoración de las 14 necesidades básicas según el modelo de Virginia Henderson, donde se puede observar lo siguiente:

- Respiración: el paciente respira con normalidad, presenta un buen patrón respiratorio.

- Ingesta hídrica y alimenticia: presenta piel y mucosas bien hidratadas, nos indica que aproximadamente tiene una ingesta hídrica de $1200 \mathrm{ml}$ de agua al día. Presenta buen patrón de alimentación, realiza 5 comidas al día y en casa se le controla la ingesta de sal en las comidas.

- Eliminación: refiere continencia tanto urinaria como fecal.

- Movimiento y mantenimiento de una postura adecuada: José Antonio es independiente para las AVD, deambula con la ayuda de un bastón por seguridad.

- Sueño/descanso: por las noches suele tener períodos de vigilia o de dormir a intervalos.

- Selección de vestimenta adecuada: se viste solo y no necesita ayuda para vestirse.

- Mantenimiento de temperatura corporal: la temperatura corporal se encuentra mantenida; su familia refiere que no han notado fiebre ni febrícula en los últimos días.

- Mantenimiento de la higiene corporal: el paciente se asea solo y, debido a eso, su familia desconocía la lesión que éste tiene en la zona mamaria derecha. Por dicho motivo presenta un deterioro de la integridad tisular.

- Evitar los peligros de su entorno: su hijo ha adaptado la casa para que pueda deambular sin problema, así como el cuarto de baño para que pueda realizar las AVD con la mayor independencia.

- Comunicación: tiene buena relación con su hijo y su nuera, expresa emociones y recuerdos con ellos.

- Ejerce culto a Dios: refieren ser creyentes aunque no practicantes.

- Trabaja de forma con la que sentirse realizado: colabora con algunas de las tareas domésticas.

- Descubre, estudia y conoce: le gusta leer el periódico cada mañana. Se establecen diferentes escalas de valoración de la salud de nuestro paciente (tabla 1).

Descripción de la herida: José Antonio presenta una herida de $7 \mathrm{~cm}$ de diámetro y $4 \mathrm{~cm}$ de grosor en su mama derecha, que está sangrando, con inflamación generalizada. Se aprecia un cambio en la coloración cutánea de la lesión con aumento de la trama vascular y zonas ulceradas; presenta la piel que recubre la herida seca y macerada aparentemente, con rotura de la epidermis donde se aprecia sangrado activo (Figs. 1 y 2); a la exploración se aprecia una tumoración que ocupa toda la mama derecha. Nos encontramos ante una tumoración nodular con consistencia pétrea y que infiltra tejidos adyacentes (según valoración por el Servicio de Cirugía).

Se establece un plan de cuidados individualizado, adaptado a los horarios de su domicilio. En función de la valoración realizada se establecen los siguientes objetivos terapéuticos: evitar la posible infección bacteriana y manejo de la hemorragia.

Se elige como principal problema, la siguiente etiqueta diagnóstica:

Diagnóstico de Enfermería (18) 00046: deterioro de la integridad cutánea, relacionado con cambios en la pigmentación, y deterioro de la sensibilidad, manifestado por destrucción de las capas de la piel.

Entre los principales criterios de resultados (NOC) perseguidos en este caso nos encontramos con (19):

NOC: curación de la herida: por segunda intención (1103) (v. indicadores en tabla 2), del que se derivan las intervenciones de enfermería (NIC) especificadas en la tabla 3, así como sus actividades de enfermería relacionadas (20): cuidados de las heridas y control de infecciones.

En el informe de alta de enfermería se especifica, además del diagnóstico, resultados e intervenciones realizadas, diferentes pautas de actuación, educación para la salud relacionada y cuidados locales

Tabla 2. Distribución de puntuaciones del criterio de resultado Curación de la herida (NOC) en el momento de la atención

Curación de la herida: por segunda intención (1103)

\section{Indicadores}

Valoración

(110320) Formación de cicatriz

Ninguna

(110321) Disminución del tamaño de la herida

(110305) Secreción sanguinolenta

Ninguna

(110307) Eritema cutáneo circundante

Moderada

(110307) Eritema cutáneo circundante

Sustancial

(110322) Inflamación de la herida

Extensa

(110308) Edema perilesional

Moderado

(110317) Olor de la herida

Moderado

Resto de indicadores no aplicables. 
Tabla 3. Distribución de las intervenciones de enfermería y actividades relacionadas para el manejo de la herida

\begin{tabular}{|l|l|l|}
\hline Código & NIC & Actividades \\
\hline & 366030 Documentar la localización, tamaño y apariencia de la herida \\
& 366029 Enseñar al paciente y la familia los signos y síntomas de infección \\
\hline \multirow{3}{*}{3660} & Cuidados de las heridas & 366017 Comparar y registrar regularmente cualquier cambio producido en la herida \\
& 366015 Cambiar el apósito según la cantidad de exudado y drenaje \\
& 366007 Limpiar con solución salina normal o limpiador no tóxico, si procede \\
& 366003 Controlar las características de la herida incluyendo drenaje, color, tamaño y olor \\
\hline \multirow{3}{*}{6540} & 654024 Asegurar una técnica de cuidados de herida adecuada \\
\hline \multirow{3}{*}{ Control de infecciones } & 654013 Poner en práctica precauciones universales \\
& & 654035 Enseñar al paciente y a la familia a evitar infecciones \\
\hline
\end{tabular}

de la herida, tanto al paciente como a sus familiares. Se insiste en el cuidado de la piel perilesional; principalmente, mantener la piel limpia y seca y evitar la fricción. Se recomienda usar agua tibia y jabones neutros, poco irritantes, así como desinfección con povidona yodada o clorhexidina.

Se les indica que han de pedir cita con su enfermera de atención primaria, para control y seguimiento de la herida. Además, se explica a la familia la importancia del control de la fiebre, del tipo de exudado que tenga la herida, del control de nuevas hemorragias, de detectar si presenta mal olor, la importancia del control de la infección, así como el riesgo de padecerla y de ser así acudir a su centro de atención primaria.

Tras la valoración inicial en urgencias y administradas las primeras curas, el médico encargado de valorar al paciente lo remite a la consulta específica de cáncer de mama. Allí se reevalúa al paciente al cabo de un mes y se decide la realización de una biopsia de la lesión, así como la práctica de un estudio de extensión, de cara a valorar los siguientes pasos a seguir en el tratamiento de este paciente.

Dado el estadio de su enfermedad en el momento del diagnóstico y la patología de base del paciente, se decide optar por un tratamiento paliativo. Se explica a la familia la importancia de realizar a los hijos del paciente un estudio genético, para detectar si existe algún factor que pueda ser hereditario y pueda causar en el hijo la misma patología.

\section{O DISCUSIÓN}

José Antonio fue dado de alta a su domicilio y derivado a la consulta específica de cáncer de mama. No requirió tratamiento quirúrgico. No debemos olvidar que los pacientes que se encuentran en una etapa terminal, donde no existe una cura posible, tienen la opción de hacer uso de los cuidados paliativos. Estos cuidados son ofrecidos y van dirigidos a preservar la mayor calidad de vida posible a este tipo de pacientes y a sus familiares, ya que es ésta, la familia, quien se convierte en la principal cuidadora; ellos ponen en conjunto el afecto, los conocimientos que adquieren durante este proceso del personal sanitario y su fuerza de voluntad, los que hacen que el paciente con cáncer terminal tenga la opción de pasar la mayor parte del proceso de su enfermedad en su domicilio. Cabe recordar la importancia de la valoración del dolor por parte del personal de enfermería, mediante la escala visual analógica. De esta manera, se puede mantener un control y manejo del dolor en el domicilio, en caso de que el paciente empezara a notar dolor. Para el paciente, encontrarse en su hogar supone estar en un ambiente conocido, manteniendo su intimidad, para así poder realizar ciertas tareas laborales, continuar con sus hábitos y algunas de sus actividades preferidas. Es necesario también incluir a la familia del paciente en el cuidado domiciliario, que sea conocedora de las posibles complicaciones que puedan suceder, así como explicar los cuidados locales de la herida

\section{BIBLIOGRAFÍA}

1. Giordano SH, Cohen DS, Buzdar AU. Breast cancer in men. Ann Intern Med 2002; 137: 678-87.

2. Korde LA, Zujewski JA, Kamin L, Giordano S, Domchek S, Anderson WF y cols. Multidisciplinary meeting on male breast cancer: summary and research recommendations. J Clin Oncol 2010; 28: 2114-22.

3. Gui G. Male breast cancer: aetiology and clinical features. Trends in Urology \& Men's Health 2012; 3: 29-31.

4. Czene K, Bergqvist J, Hall P, Bergh J. How to trate male breast cancer. Breast 2007; 16 (Suppl 2): S147-54.

5. Giordano SH. A review of the diagnosis and management of male breast cancer. The Oncologist 2005; 10: 471-9.

6. Simon MS, McKnight E, Schwartz A, Martino S, Swanson GM. Racial differences in cancer of the male breast-15 year experience in the Detroit metropolitan area. Breast Cancer Res Treat 1992: 21: 55-62.

7. Sasco AJ, Lowenfels AB, Passer-de-Jong P. Epidemiology of male breast cancer. A meta-analysis of published case-control studies and discussion of selected aetiological factors. Int J Cancer 1993: 53: 538-49.

8. Hultborn R, Hanson C, Kopf I, Verbiene I, Warnhammar E, Weimarck A. Prevalence of Klinefelter's syndrome in male breast cancer patients. Anticancer Res 1997; 17: 4293.
9. Hsing AW, McLaughlin JK, Cocco P, Co Chien HT, Fraumeni JF Jr. Risk factors for male breast cancer (United States). Cancer Causes Control 1998; 9: 269-75

10. Ewertz M, Holmberg L, Tretli S, Pedersen BV, Kristensen A. Risk factors for male breast cancer. A case-control study from Scandinavia. Acta Oncol 2001; 40: 467-71

11. Burstein HJ, Harris JR, Morrow M. Malignant tumors of the breast. En: De Vita VT Jr, Lawrence TS, Rosenberg SA, editores. Cancer. Principles and Practice of Oncology. 9. ${ }^{\mathrm{a}}$ ed. Philadelphia: Lippincott Williams \& Wilkins, 2011, pp. 1401-46.

12. Koc M, Öztas S, Erem MT, Ciftcioglu MA, Onuk MD. Invasive lobular carcinoma of the male breast: a case report. Jpn J Oncol 2001; 31: 444-6.

13. Magro G. Mammary myofibroblastoma: a tumor with a wide morphologic spectrum. Arch Pathol Lab Med 2008; 132: 1813-20.

14. Magro G, Bisceglia M, Michal M, Eusebi V. Spindle cell lipoma-like tumor, solitary fibrous tumor and myofibroblastoma of the breast: a clinic-pathological analysis of 13 cases in favor of a unifying histogenetic concept. Virchows Arch 2002; 440 : 249-60.
15. Rosen P. Rosen's Breast Pathology. Philadelphia: Lippincott Williams \& Wilkins, 2009, pp. 829-901.

16. Borgen PI, Wong GY, Vlamis V. Current management of male breast cancer. A review of 104 cases. Ann Surg 1992; 215: 451-7.

17. Kinne DW. Management of male breast cancer. Oncology (Huntingt) 1991; 5: 45-7.

18. North American Nursing Diagnosis Association. Diagnósticos Enfermeros: Definiciones y Clasificación 2009-2011. Madrid: Elsevier, 2009.

19. Moorhead S, Johnson M, Maas M. Proyecto de Resultados lowa. Clasificación de resultados de Enfermería (NOC). 3. ${ }^{\text {a }}$ ed. Madrid Elsevier, 2005.

20. McCloskey JC, Bulecheck GM. Clasificación de Intervenciones de Enfermería (CIE). $5{ }^{\text {a }}$ ed. Madrid: Mosby, 2009.

21. Vila Arias JM. Utilidad del Palliative Performance Scale v2 para la estimación de supervivencia en enfermos con cáncer avanzado. MEDIPAL 2012: 19: 133-8. [Consultado el 14 de noviembre de 2012]. Disponible en: http://zl.elsevier.es/es/ revista/medicina-paliativa-337/utilidad-palliative-performance-scale-v2-estimacion-supervivencia-90160110-originales-2012 